\title{
Outcome of Post-Operative Mastoid Cavity Obliteration with Autologous Adipose Tissue
}

\author{
https://doi.org/10.47210/bjohns.2020.v28i2.331
}

Rabi Hembrom, ${ }^{1}$ Satadal Mondal, ${ }^{2}$ Indranil Sen, ${ }^{1}$ Amit Chakrabarti, ${ }^{1}$ Rupam Sinha, ${ }^{3}$ Tapas Kumar Mahata, ${ }^{1}$ Aritra Bhattacharya, ${ }^{1}$ Swagato Roy $^{1}$

\begin{abstract}
$\underline{\text { Introduction }}$ obliteration with autologous adipose tissue.

Materials and Methods canal wall down Mastoidectomy followed by obliteration with autologous adipose tissue in Group B

$\underline{\text { Results }}$ weeks.

$\underline{\text { Conclusion }}$

Cavity problems encountered is considerably less in the group obliterated with adipose tissue.

$\underline{\text { Kevwords }}$

Mastoidectomy; Adipose Tissue
\end{abstract}

$\underline{\text { ABSTRACT }}$

Mastoid operations have been in practice for over four centuries for suppurative conditions of the ear. Intact canal wall mastoidectomy has the advantage of better functional results while canal wall down mastoidectomy offers excellent exposure for disease eradication and post operative monitoring but is associated with significant cavity problems. In order to overcome the problems associated with canal wall down procedure while retaining its advantages the concept of mastoid cavity obliteration was introduced. This study analysed the outcomes of mastoid cavity obliteration and to assess the outcomes of mastoid cavity

A prospective, experimental, randomized study was conducted over a period of 18 months among patients presenting with active squamous variety of Chronic Otitis Media. The patients were randomly allocated to two groups, $A$ and B. Both groups underwent

In group A, the mean duration required for complete epithelialization was 10.8 weeks. In group B, the average time taken for complete epithelialization was 5.6 weeks. All cases had their graft intact at the end of 12 weeks. Debris was present in group A for a mean duration of 9.47 weeks. In group B, debris was found for a mean duration of 3.33 weeks. Patients from group A complained of discharge from their ears for a mean duration of 7.47 weeks. In group B the same symptom persisted over 3.33

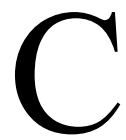
hronic otitis media is an inflammatory process in the middle-ear space that results in longterm, or more often, permanent changes in the tympanic membrane including atelectasis, dimer

1 - Department of Otorhinolaryngology, Midnapore Medical College, Vidyasagar Road, Midnapore

2 - Department of Otorhinolaryngology, I.P.G.M.E.R.,

Kolkata

3 - Khatra Sub Division Hospital, Bankura

Corresponding author:

Dr Amit Chakrabarti

email: amitchakrabarti77@gmail.com formation, perforation, tympanosclerosis, retraction pocket development or cholesteatoma. Nadol classified chronic otitis media as chronic active otitis media with or without cholesteatoma; chronic inactive otitis media with perforation, with retraction pocket, adhesive otitis media, with ossicular fixation or resorption; chronic inactive otitis media with frequent reactivation. ${ }^{1,2}$ Another proposed classification is based on classifying the disease into mucosal(active/inactive); squamous(active/ inactive) and healed. ${ }^{3}$

Mastoid operations have been in practice for over four centuries for suppurative conditions of the ear. Jansen 
described canal wall up mastoidectomy in 1953. The main advantage of canal wall up mastoidectomy is that normal anatomy can be preserved along with clearance of disease process. ${ }^{4}$

But in cases of unresectable cholesteatomas extending up to petrous apex, unreconstructible posterior canal wall, failure of first stage canal wall up procedures owing to poor Eustachian tube function, a canal wall down mastoidectomy is done which requires the removal of posterior wall of external auditory canal. ${ }^{5}$

Intact canal wall mastoidectomy has the advantage of rapid wound healing and also avoids frequent cleaning of the cavity. But the chances of residual disease as well as recurrent cholesteatoma is high with this procedure. ${ }^{6}$

With canal wall down mastoidectomy there is excellent exposure for disease eradication and post operative monitoring; also there is less chance of leaving behind residual disease with low rates of recurrence. ${ }^{7}$ But it is associated with patient's intolerance to water exposure; requirement of frequent cleaning of cavity; calorically and barometrically induced vertigo; aesthetic inconvenience due to too large meatoplasty; recurrent infection; difficulty in wearing traditional hearing aids. Also the final hearing gained after staged ossiculoplasties in patients who have undergone canal wall down mastoidectomy is usually $5-10 \mathrm{~dB}$ worse than those who underwent canal wall up tympano-mastoidectomy due to ineffective sound transmission. ${ }^{6,78.9}$

In order to overcome the problems associated with canal wall down procedure while retaining its advantages the concept of mastoid cavity obliteration was introduced by Mosher in $1911 .^{6}$ Over the course of this century, there have been numerous reports detailing a variety of techniques of obliterating the mastoid cavity.

In developing countries like India where proper health care services is still not available to a sizeable chunk of the society, mastoid obliteration reduces the number of hospital visits for cavity management.

Keeping this in mind, the following study has been conducted with the aims and objectives to study the outcomes of mastoid cavity obliteration and to assess the outcomes of mastoid cavity obliteration with autologous adipose tissue as obliterating material for mastoid obliteration.

\section{Materials and Methods}

A prospective, experimental, randomized study was conducted over a period of 18 months amongst the patients attending the Otorhinolaryngology out-patients wing of a peripheral referral institute from January 2017 to June 2018. All cases of chronic otitis media with cholesteatoma, with extensive granulations, with attic or postero-superior retraction pockets where the fundus is not visible were included in the study. Known cases of chronic otitis media with complications and those who didn't wish to participate in the study were excluded.

After proper selection, the patients were counselled about the disease process and the treatment modalities with the expected outcome and the complications of the procedures. They were then subjected to pure tone audiometry, X-ray mastoids, Examination under microscope, routine pre operative investigations followed by pre anaesthetic check-up. The patients were then divided into two groups by systematic random sampling-

Group-A: Canal Wall Down (CWD) Mastoidectomy without obliteration

Group-B: CWD Mastoidectomy and obliteration with adipose

Operative procedure: All the surgeries were done under general anaesthesia. Lignocaine $(2 \%)$ with 1:100000 adrenaline was infiltrated for haemostasis. Temporalis fascia graft was harvested. Post auricular incision was made. A Canal Wall Down mastoidectomy was done to remove any disease process in the mastoid and middle ear. It was followed by ossicular reconstruction if required, followed by tympanoplasty. Then, according to pre-determined protocol, the mastoid cavity was left as such (Group A) or obliterated using autologous adipose tissue (Group B). (Fig. 1)

Post operative treatment and follow up: Each patient was given an antibiotic, analgesic and antihistaminic medication for one week. Patients were discharged on the second postoperative day. Ear pack was removed two days after surgery. Patients were then asked to follow-up at two weeks after pack removal, one and half months post-operative and 3 months postoperative for assessment of hearing by pure tone audiogram. 


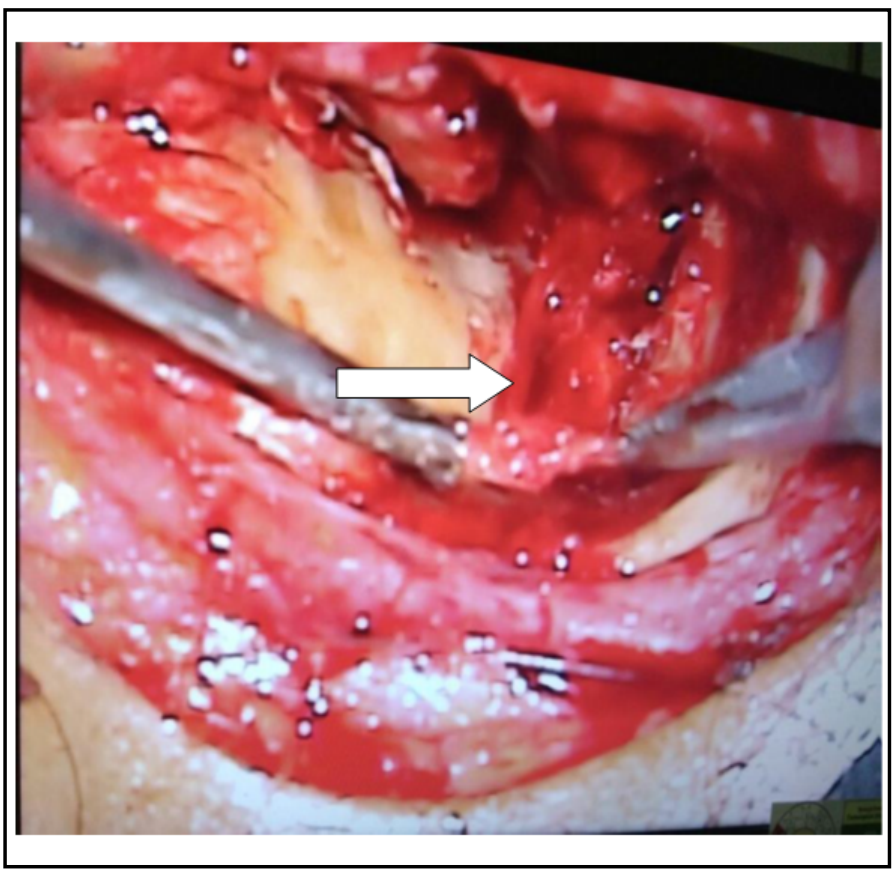

Fig. 1. Intra-operative picture showing Canal Wall Down Mastoidectomy with obliteration with adipose tissue

\section{Results}

The impact of the surgical procedures undertaken were assessed on the basis of (a) healing of mastoid cavity in terms of epithelialisation, (b) post-operative problems and (c) hearing gain.

Each group comprised of 15 individuals. The individuals were divided into 4 age groups: $5-15$ years, 16-25 years, 26-35 years and 36-45 years. A total of 9 males and 6 females were in group A, 7 males and 8 females in group B. The mean age of the individuals in group A was 25.47 years with a standard deviation of 8.89 years; in group B it was 24.4 years with a standard deviation of 10.13 years.

The individuals in each group underwent Examination under microscope (EUM) prior to surgery. Individuals with attic pathology comprised the majority (5 cases in each group) followed by postero-superior retraction pockets (PSRP) and adhesive otitis media.

Conductive deafness was prevalent in both the groups with mixed deafness detected only in 2 patients in group A and 1 in group B.

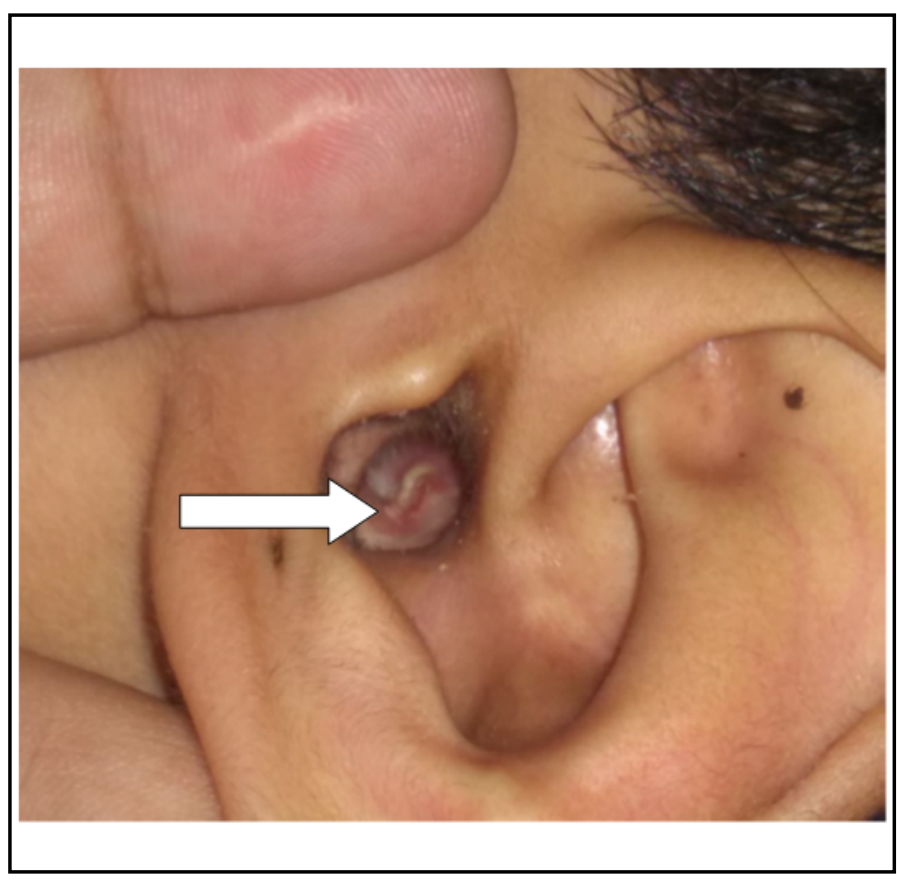

Fig. 2. Post operative photograph showing a well epithelialized, obliterated mastoid cavity at 12 th week

The pre-operative mean pure tone average in group A was $47.6 \mathrm{~dB}$ with a standard deviation of $10.34 \mathrm{~dB}$. The mean Air-bone gap (A-B gap) in group A was 25.87 $\mathrm{dB}$ with a standard deviation of $9.99 \mathrm{~dB}$. The mean pure tone average in group $\mathrm{B}$ was $46.6 \mathrm{~dB}$ with a standard deviation of $10.81 \mathrm{~dB}$. The mean A-B gap in group B was $25.93 \mathrm{~dB}$ with a standard deviation of $9.6 \mathrm{~dB}$. In both groups, individuals mostly had moderate degree of hearing loss followed closely by individuals with mild degree of loss ( 9 moderate and 4 mild in group A; 10 moderate and 4 mild in group B).

On classifying the study subjects based on the type of tympanoplasty, Type III tympanoplasty was most commonly performed in both the groups followed by type IV tympanoplasty.

The study subjects have been followed up in the post operative period according to the protocol as previously determined. Examination under microscope was carried out during each post-operative visit. The following parameters were noted, namely, epithelialization of the mastoid cavity; presence of debris in the mastoid cavity; duration of otorrhoea; common postoperative complications like pain, vertigo, granulation tissue, 
morbidity of donor site etc; graft integrity at postoperative visits of 2 weeks, 4 weeks, 6 weeks and 12 weeks. The post-operative pure tone audiometry was performed at 12 weeks and the hearing outcome evaluated based on A-B gap closure.

Epithelialization of the mastoid cavity was noted as partial or complete during each of the post-operative visits. In group $\mathrm{A}$, the mean duration required for complete epithelialization was 10.8 weeks with a standard deviation of 2.48 weeks. In group B, the average time taken for complete epithelialization was 5.6 weeks with a standard deviation of 2.03 weeks. The time taken for epithelialization amongst the 2 groups have been assessed with unpaired t-test and it has been found that the difference in time taken for epithelialization is highly significant when group A (CWD Mastoidectomy without obliteration) is compared against group B (CWD Mastoidectomy with obliteration with adipose). (Fig. 2)

As far as graft integrity is concerned, all cases had their graft intact at the end of 12 weeks. Post-operative pain was complained by 4 patients in the group without obliteration; 3 in case of obliteration with adipose. There was no further complaint during the study period. Presence of debris in the mastoid cavity was then evaluated amongst the groups. Debris was present in group A for a mean duration of 9.47 weeks with a standard deviation of 3.25 weeks. In group B, it has been found for a mean duration of 3.33 weeks with a standard deviation of 1.23 weeks. Collection of debris in the mastoid cavity has been assessed using the same unpaired t-test as above with similar statistical significance being obtained between obliterated versus unobliterated group.

Vertigo in the post-operative period was complained mainly by patients in the group which did not undergo any obliteration. 8 cases complained of vertigo in the immediate 2nd week of post-operative visit, of which 5 cases had their symptoms resolved by the end of 4 weeks whereas another 2 individuals had persistent symptoms till the end of 6 weeks. By the end of 12 weeks no one complained of vertigo. In group B only 1 patient complained of vertigo at 2 nd week which resolved subsequently with no such complaints during any of the subsequent post-operative visits.
Otorrhoea following canal wall down procedures being the most frequent complaint during the postoperative period has been evaluated next. Patients from group A complained of discharge from their ears for a mean duration of 7.47 weeks with a standard deviation of 2.88 weeks. In group B the same symptom persisted over 3.33 weeks with a standard deviation of 1.23 weeks. The duration of otorrhoea was evaluated using the same principles with no different statistical outcome, i.e., obliteration groups showing better results compared to group A.

At the end of 12 th post-operative week, all individuals were subjected to pure tone audiogram. In group $\mathrm{A}$, the mean PTA was $36.06 \mathrm{~dB}$ with a standard deviation of $10.05 \mathrm{~dB}$. The mean A-B gap in group A was 17.07 $\mathrm{dB}$ with a standard deviation of $10.59 \mathrm{~dB}$. In group $\mathrm{B}$, the mean PTA was $36.2 \mathrm{~dB}$ with a standard deviation of $10.42 \mathrm{~dB}$. The mean A-B gap in group B was 15.2 $\mathrm{dB}$ with a standard deviation of $10.06 \mathrm{~dB}$. Each group showed statistically significant improvement in pre and post-operative pure tone audiometry and A-B gap when tested by paired t-test but the hearing gain in the individual groups when tested against each other failed to produce any statistical significance when tested by unpaired t-test.

\section{Discussion}

The global burden of illness from COM involves 65-330 million individuals with draining ears, $60 \%$ of whom (39200 million) suffer from significant hearing impairment. Over $90 \%$ of the burden is borne by countries in the south-east Asia and western Pacific regions, Africa, and several ethnic minorities in the Pacific rim. COM is uncommon in the Americas, Europe, the Middle East, and Australia. Among the South-East Asian countries, prevalence rate of COM in Thailand ranged from 0.9 to $4.7 \%$ while the Indian prevalence is $7.8 \%{ }^{10}$

The primary goal of middle ear surgery, whether or not the patient has a cholesteatoma, is extirpation of the disease, anatomical preservation of the middle ear, and functional restoration. ${ }^{11}$ The type of mastoidectomy is based on the extent of the disease, preoperative health of the patient, the status of the opposite ear, and both the surgeon's and patient's preference. ${ }^{10}$ 
The canal wall up mastoidectomy involves removing the mastoid air cells lateral to the facial nerve and otic capsule bone while preserving the posterior and superior external auditory canal walls. It preserves the normal anatomy of the ear canal, thus avoiding the risk of bowl problems and the necessity for periodic cleaning, leading to reduced healing time and, possibly, better hearing results. However, a high residual and recurrence rate continue to be major drawbacks to canal wall up surgery. ${ }^{10}$

The canal wall down technique creates an open cavity after removal of the bony posterior canal wall, providing excellent exposure for cholesteatoma removal. Recurrence rate is lowered to $5-10 \%$. The main disadvantages of this technique are related to the necessity for periodic cleaning of the mastoid cavity with persistent otorrhea. Other problems with open cavity include difficulty in the use of a hearing aid, water intolerance due to susceptibility to infection, and proneness to vertigo by a caloric stimulus such as warm/cold air or water. CWD when compared to CWU has lower risk of recurrence (2-10\%) but higher risk of otorrhoea (20-60\%). These problems can be minimized by different obliterative procedures. ${ }^{10}$

The concept of mastoid cavity obliteration was first introduced by Mosher in 1911. Mastoid reconstruction and obliteration procedures involve free grafts (which are further sub-divided to biologic and non-biologic) and local flaps.

With the advent of numerous techniques for mastoid obliteration, the debate continues till date regarding the best material that can be used for obliteration. Each method has some distinct benefits over the others. Although these techniques offer the major advantage of eliminating the cavity problem, there can be long-term problems. For example, some of the obliterated tissues can shrink over time, minimizing the effectiveness of the mastoid obliteration. ${ }^{11}$

Obliteration with fat has been of particular interest due its inherent properties, which may be the cause for low complication rate associated with use of fat. Fat tissue is not inert; it stores macrophages, which participate in inflammation and the immune reaction. Fat tissue also stores a high proportion of lymphocytes which, along with macrophages, can lead to immune regulation. Also fat provides a ready contrast medium in postoperative fat suppression and diffusion sequences on the MRI scan, which provides a very adequate method to detect recurrence. $^{12}$

The mean age of the individuals in our study- in group A was 25.47 years with a standard deviation of 8.89 years; in group B it was 24.4 years with a standard deviation of 10.13 years. The mean age of individuals in the study by Bhandary et al. was $26.95+9.525$ years which is coinciding with our study. ${ }^{13}$

On examination under the microscope, $62 \%$ subjects in our study had cholesteatoma (comprising both PSRP with cholesteatoma as well as attic cholesteatoma), nearly $9 \%$ had granulation and $29 \%$ comprised the remainder. In the study by Deshmukh et al, $80 \%$ of patients had cholesteatoma, $13.3 \%$ had granulations and $6.6 \%$ comprised the remainder. ${ }^{14}$ The findings of our study are in tune with the above study where individuals with cholesteatoma formed the majority followed by granulation tissue.

In the present study nearly $89 \%$ individuals had mildmoderate degree of hearing loss. This finding is slightly different from that of the study mentioned above, where $33.3 \%$ had hearing loss of $<30 \mathrm{~dB}, 50 \%$ had hearing loss between 30 and $60 \mathrm{~dB}$ and only $16.6 \%$ had hearing loss $>60 \mathrm{~dB} \cdot{ }^{14}$

The mean time required for complete epithelialization of un-obliterated mastoid cavities in this study was around 3-6 months which correlates well with that of Deshmukh et al where $94 \%$ cases showed epithelialization at the end of 6 months. ${ }^{14}$ The mean duration for complete epithelialization of cavities obliterated by adipose tissue was 6-8 weeks in the present study. This correlates with the study conducted by Ravi and Kumar ${ }^{15}$ but does not agree with the study by Bhandary et $a{ }^{13}$ where the mean duration was around 17 weeks.

The time required to obtain a dry cavity in unobliterated cases was around 8-10 weeks in our study which correlates well with the study by Desmukh et al. ${ }^{14}$ In cases obliterated with adipose tissue the time required to obtain a dry cavity was around 4-5 weeks in our study. None of the other studies (Ravi and Kumar, ${ }^{15} \mathrm{P}$. Montandon et all ${ }^{\circ}$ ) had any other complaints regarding 
otorrhoea.

Post-operative vertigo was found to be significantly higher in groups that did not undergo any obliteration compared to groups that were obliterated with adipose.

Post-operative hearing was found to improve in both the study groups. However only a modest improvement in post-operative hearing is seen in studies where no obliteration was done. ${ }^{11,16}$

\section{Conclusion}

Obliteration of the mastoid cavity offers possible remedy to the various cavity problems associated with a large mastoid cavity. This study, conducted with the aim to assess the outcomes of mastoid cavity obliteration using autologous adipose tissue demonstrated shorter duration of accumulation of debris and early achievement of dry ear resulting in early healing of mastoid cavity in terms of epithelialization is obliterated cavities.Hearing gain showed no difference when compared amongst the two groups though each group showed significant statistical improvement post-operatively.

Thus, it can be seen that cavity obliteration offers a simple solution to avoid the cavity problems and also promotes early healing of mastoid cavity. Adipose being easily available, can be readily used for obliteration. However, larger studies are needed to conclusively prove or refute the hypothesis statistically.

\section{References}

1. Gopen Q. Pathology and clinical course of the inflammatory diseases of the middle ear. In: Gulya AJ, Minor LB, Poe DS, editors. Glasscock-Shambaugh Surgery of the Ear. 6th ed. Shelton, Connecticut: People's Medical Publishing HouseUSA; 2010

2. Nadol JB. The chronic draining ear. Current therapy in Otolaryngol Head Neck Surg. 1987;18:18-22

3. Browning GG. Merchant SN, Kelly G, Swan IR, Canter R, McKerrow WS. Chronic otitis media. In: Gleeson M, editor. Scott-Brown's Otorhinolaryngology, Head and Neck Surgery. 7th ed. Great Britain. Edward Arnold (Publishers) Ltd; 2008

4. Haynes DS, Wittkopf J. Canal-wall-Up Mastoidectomy. In:
Gulya AJ, Minor LB, Poe DS, editors. Glasscock-Schambaugh Surgery of the Ear. 6th ed. Shelton, Connecticut: People's Medical Publishing House-USA; 2010

5. Kveton JF. Open Cavity Mastoid Operations. In: Gulya AJ, Minor LB, Poe DS, editors. Glasscock-Schambaugh Surgery of the Ear. 6th ed. Shelton, Connecticut: People's Medical Publishing House-USA; 2010

6. Rao U.S. Obliteration of mastoid cavity and reconstruction of attic area using conchal cartilage. Asian Pac. J. Health Sci. 2016; 3(1):140-3

7. Cho SW, Cho YB, Cho HH. Mastoid Obliteration with Silicone Blocks after Canal Wall Down Mastoidectomy. Clinical and Experimental Otorhinolaryngology 2012; 5(1):23-7

8. Uçar C. Canal wall reconstruction and mastoid obliteration with composite multifractured osteoperiosteal flap. European Archives of Oto-Rhino-Laryngology 2006; 263(12):1082-6

9. Alves RD, Cabral Junior F, Fonseca AC de O, Bento RF. Mastoid Obliteration with Autologous Bone in Mastoidectomy Canal Wall Down Surgery: a Literature Overview. International Archives of Otorhinolaryngology 2016; 20(1):76-83

10. Singh M, Jain S, Rajput R, Khatua RK, Sharma D. Retrospective and Prospective Study of Singapore Swing Method on Healing of Mastoid Cavity. Indian J Otolaryngol Head Neck Surg. 2010; 62: 365-71

11. Lee HJ, Chao JR, Yeon YK, Kumar V, Park CH, Kim HJ, Lee JH. Canal Reconstruction and Mastoid Obliteration Using Floating Cartilages and Musculoperiosteal Flaps. Laryngoscope 2017 May;127(5):1153-60

12. Preetam C, Verma R, Thakar A, Sikka K. Tympanomastoid obliteration for recalcitrant attico-antral chronic suppurative otitis media. Indian J Otol. 2011;17:26-9

13. Bhandary S, Paudel D, Chettri ST, Natesh VN, Shilpakar SL, Sah BP, Pokharel A. Obliteration of Mastoid Cavity with Temporalis Muscle Pedicle and Abdominal Fat Graft and Compare the Efficacy of Each Method: A Prospective Randomized Clinical Trial. Otolaryngology Online Journal 2015; 5(3)

14. Deshmukh S, Sharma A, Dabholkar J. Mastoid cavity obliteration: Our experience. Otolaryngologia Polska 2012; 66:379-81

15. Ravi K, Kumar SK. Mastoid cavity obliteration in open cavity type (MRM) with fibro fatty tissue and subcutaneous periosteal flaps study conducted at RIMS college Ongole ENT department 2015-2016. Journal of Dental and Medical Sciences. 2016;15(6):63-70

16. Montandon P, Benchaou M, Guyot JP. Modified canal wall-up mastoidectomy with mastoid obliteration for severe chronic otitis media. ORL J Otorhinolaryngol Relat Spec. 1995; 57(4):198-201. 